J. Clifford Murray (ed)

\section{Angiogenesis Protocols}

Humana Press, Totowa 2001

USD 99.50

ISBN 0-896-03698-7

Angiogenesis research after languishing for years as a laboratory phenomenon has exploded onto the scientific and public scene. A wide variety of diseases are associated with abnormalities in angiogenesis, either deficiency or excess. What has also happened is the knowledge that a wide variety of agents, both old and new, can be shown to inhibit the many steps in angiogenesis.

This book, edited by Dr. J. Clifford Murray, continues the series on Methods of Molecular Medicine. The first chapter is a concise overview of the field. The next 16 chapters define methods for assaying angiogenesis effects in vitro and in vivo. These assays are extremely important for defining activity of the agents as well as biologic monitoring of the effects of agents in humans. As with the other books on methods, each author defines specific protocols for the assays. Extensive reference lists are given. The book is very timely and useful to the laboratory and clinical investigators who are in this area.

Paul P. Carbone, Madison, Wisc.

A.B. Miller, H. Bartsch, P. Boffetta, et al. (eds)

\section{Biomarkers in Cancer Chemoprevention}

\author{
IARC Scientific Publications, No. 154/2001 \\ Oxford University Press, Oxford 2001 \\ 294 pp.; GBP 20.00 \\ ISBN 92-832-2154-0
}

Biomarkers are meant to be indicators of exposure, intermediate and drug effects, tumor and susceptibility to cancer. These markers are intended to be short-term indicators of effect in phase II trials. Few, however, have been evaluated in phase III trials. The aim in cancer prevention trials is to uncover an intermediary marker like serum cholesterol in the prevention of myocardial infarcts.

The IARC convened a workshop in Heidelberg, Germany, in February 2000. This publication is the report of the proceedings of this workshop. Besides the individual papers, the book begins with a comprehensive report in chapter I. Thirteen recommendations summarize the outcome of the workshop. The people contributing to the book are all experts. The material is presented in many dense tables and references.

The book is an excellent reference for investigators in the field. However, with the exception of tracking the decrease of colon polyps (as they are a precursor to colon cancer), a practical demonstration of the utility of biomarkers is lacking.

Paul P. Carbone, Madison, Wisc.
J.R. Siewwert, D. Kelsen, K. Maruyama

\section{Gastric Cancer \\ Diagnosis and Treatment: An Interactive Training \\ Program}

Springer, Berlin 2000

ISBN 3-540-14787-X

This CD-ROM was developed by the Department of Surgery, Technische Universität, Munich, Germany. It attempts to provide a comprehensive interactive training program. Great effort has been made to include video clips of various surgical techniques and diagrams. It offers the user the ability to explore areas from the epidemiology and pathology of the disease to diagnostic tests and treatment options.

One drawback is that it is occasionally difficult to understand the choice of words and grammar used. Information such as survival data is skewed, with a single center in Japan providing the patient population that is the basis of calculating survival. The program has a surgical focus with the bias of a particular department towards treatment options. Certainly, much more about chemotherapy and radiotherapy could be explained, given their expanding and increasingly important roles in the treatment of gastric cancer.

This program will suit surgeons more than oncologists, given its greater emphasis on the surgical aspects of gastric cancer. The media used to convey the text and imagery varies in quality, from impressive video clips of surgery to somewhat complicated diagrams on anatomy.

Robert Lim, Singapore

\section{Winsor Bowsher, W.F. Hendry \\ Challenges in Prostate Cancer}

Blackwell, Oxford 2000

ISBN 0-632-05422-0

Prostate cancer is an international problem in developed countries. This cancer has become the most common tumor diagnosed in men. With the advent of the PSA test, more cancers are being diagnosed, more of which are thought to be clinically not significant. This book contains 16 chapters written mainly by European specialists that cover biology, diagnosis, treatment, and prevention. The book contains little new information, but it does present the assessment by specialists who are able to add a degree of neutralism, sometimes not seen by the writings of the advocates.

The first chapter starts off with a discussion of diet and prostate cancer risk. The focus is on nonsteroidal weak estrogens found in plants and plant products and how they may explain geographic differences in incidence. The author of the chapter that discusses PSA

\section{KARGER}

Fax +41613061234 E-Mail karger@karger.ch www. karger.com

\section{(C) 2001 S. Karger AG, Basel}

Accessible online at:

www. karger.com/journals/ocl 
screening obviously is not convinced of its utility. A thorough review of the EORTC trials is given in the third chapter. A deficiency in the book involves the use of radiotherapy in the management of prostate cancer. One chapter discusses the role of radiotherapy in T3 disease only and a chapter late in the book talks about interstitial therapy. The chapter was one of the few written by an American. There are several interesting chapters on cost analyses and sexual dysfunction management. Those reflect the more conservative approach of many 'do no harm'. The Europeans have generally been more willing to look at these aspects of cancer management.

The book serves an important counterpoint to those books and articles written by the advocates for one approach or another. It is thorough in its assessments, although clearly it does not cover the entire spectrum of the disease. It is written clearly and is easy to read for a non-specialist. The illustrations and charts add to the clarity. The book is a very good addition to the library of those who see prostate cancer patients.

Paul P. Carbone, Madison, Wisc.

William R. Miller, Richard J. Santen

\section{Aromatase Inhibition and Breast Cancer}

Marcel Dekker, New York 2000

309 pp.; USD 150.00

ISBN 0-8247-0412-6

Interest in aromatasase inhibitors, as a treatment option for breast cancer, has recently been heightened by the development of new drugs that are more potent and less toxic. Studies with these newer drugs have indicated that they may be useful as primary therapy because they seem to have effectiveness equivalent to tamoxifen. Miller and Santen have compiled a new book dedicated to these agents from a meeting held on the subject. This book covers the field from the history of the agents to the management of primary as well as recurrent breast cancer. Several chapters discuss the chemoprevention possibilities of these agents. More than a third of the book focuses on new indications including their use on prostate cancer, pubertal gynecomastia and endometriosis. An intriguing aspect of the book is the detailed publication of panel discussions conducted at the meeting. The questions and answers are there to review. Many of the questions revolve around practical issues.

The book is a nice account of the meeting, probably supported by pharmaceutical companies. However, little mention is made of any possible conflicts that might be created by support of the investigators or trials for these industries. Another issue is the time delay between the meetings and publication. Each panel discussion is clearly marked by a date, November 12, 1999. The data are obviously dated. However, the book has some value as a historical record of the development of these agents. Some attempt should have been made to update the presentation.

Paul P. Carbone, Madison, Wisc.
Stanley R. Hamilton, Lauri A. Aaltonen (eds)

\section{World Health Organization Classification of Tumours \\ Pathology and Genetics of Tumours of the Digestive System}

IARC Scientific Publications

Oxford University Press, Oxford 2000

314 pp.; GBP 46.00

ISBN 92-832-2410-8

Gastrointestinal cancers represent one of the common cancers in both males and females. The long intestinal tract and associated organs present multiple opportunities to initiate neoplastic processes. This book by Hamilton and Aaltonen is a gem. Not only have they managed to incorporate many histological as well as clinical photographs, but they also include the latest information on genetic changes. The clinical notes also add to the comprehensiveness of the book. The book is divided into chapters based on the gastrointestinal tract and the associated organs such as the liver, gall bladder, and pancreas. As a result, tumors such as the lymphomas get repeated, although the information in each chapter relates to that particular organ or tissue.

This book is a tremendous advance from the fascicle produced by the US Armed Forces Institute. It will serve the students and experts well.

Paul P. Carbone, Madison, Wisc.

\author{
Zbigniew Petrovich, Luther W. Brady, Michael L.J. Apuzzo, \\ Michael Bamberg (eds)
}

\section{Combined Modality Therapy of Central Nervous System Tumors}

Springer, Berlin 2001

640 pp.; 391 fig.; 80 tab.

DEM 498.- / ATS 3,636 / CHF 429.- / GBP 171.50 / USD 250.00

ISBN 3-540-66053-4

Although relatively uncommon in comparison to malignancies of the lung, breast, prostate and colon/rectum, CNS tumors pose a major oncologic change because they frequently afflict the young and have a major impact on functional outcome. Historically, surgery and radiotherapy have formed the mainstay of therapy for these patients. An emerging body of data is beginning to identify the role of chemotherapy, and in particular, recent randomized trials are beginning to better define appropriate combination approaches for various CNS tumors. This subject is the primary theme addressed in a next text, 'Combined Modality Therapy of Central Nervous System Tumors' edited by Brady, Heilmann and Molls. The book is well written and will form a useful addition for neurosurgeons, radiation and medical oncologists and neuro-oncologists. The organizational structure of the text is easy to follow, with preliminary key chapters on epidemiology, pathology, molecular biology, general therapeutic principles, introductory radiation therapy physics and imaging. Subsequent disease-specific chapters address several major primary CNS tumors, including pituitary adenoma, craniopharyngioma, vestibular schwannoma, meningioma, low grade glioma, malignant glioma, pri- 
mary CNS lymphoma, brain metastases and pediatric tumors. The closing chapters address evolving areas such as radiosurgery, immunotherapy, gene therapy, toxicities, quality of life and nursing considerations.

In general, the text is succinct, well written, thoroughly illustrated and enhanced by the liberal use of tables and figures. Since several phase III randomized trials of combination therapy have only recently become available in abstract form at various national meetings, it has not been possible to include these in the current textbook. In spite of this limitation, the book is recommended as a well-written and thorough text.

Minesh P. Mehta, Madison, Wisc.

\section{Scarpignato (ed)}

\section{Somatostatin Analogs in Cancer Management}

Reprint of 'Chemotherapy', vol 47, suppl. 2

Karger, Basel 2001

198 pp.; CHF 112.00 / DEM 145.00 / USD 97.50

ISBN 3-8055-7035X

This supplement of the journal, Chemotherapy contains 11 monographs on the biology, chemistry and application of somatostatin analogs in the management and diagnosis of cancer. Somatostatin has an important role in regulating the function of many organs, including brain, pituitary, gastrointestinal tract, pancreas and lymphoid cells. It acts as an inhibitory factor preventing the overstimulation of these organs by many growth factors. In addition, it has a direct effect on the growth of both cancer and normal cells. In experimental animals, it has a significant anti-tumor effect on many rodent tumors and transplantable human cancer cells. It can also improve symptoms such as diarrhea and flushing. While the natural form of somatostatin has a short half-life, analogs with longer effective durations of actions have made it possible to treat human diseases. Radiolabeling the somatostatin analogs has made them useful as imaging agents in defining sites of certain neuroendocrine tumors.

At higher doses, a few patients have had significant anti-tumor effects, but in general the tumors have usually failed to diminish on treatment. The book, written by experts in the field, deals with the many potential and current uses of somatostatin analogs in neuroendocrine tumors, e.g. carcinoids, gastrinomas, and VIPomas. Other chapters deal with the rather minimal anti-tumor effects in breast, lung, prostate, pancreas, liver and biliary tract tumors. There is a repetitive theme indicating the need to use this agent earlier in the disease course and in combination with other agents. The rationale of looking for analogs that effect the other five somatostatin receptors is presented. This book is an excellent reference text for those who are interested in the now fashionable molecular approaches to cancer therapy using specific targets.

Paul P. Carbone, Madison, Wisc. 